\title{
Transesophageal needle aspiration using a third-generation Olympus ultrasound bronchoscope for subaortic lesions: a report of two cases
}

\author{
Masahide Oki, Hideo Saka, Yoshihito Kogure \\ Department of Respiratory Medicine, National Hospital Organization Nagoya Medical Center, Nagoya, Japan \\ Correspondence to: Masahide Oki, MD. Department of Respiratory Medicine, National Hospital Organization Nagoya Medical Center, 4-1-1 \\ Sannomaru, Naka-ku Nagoya 460-0001, Japan. Email: masahideo@aol.com.
}

\begin{abstract}
Transesophageal endoscopic ultrasound with bronchoscope-guided fine needle aspiration (EUSB-FNA) has been reported to be useful for evaluating paraesophageal lesions; however, its ability to access subaortic lesions is relatively limited. The third-generation Olympus ultrasound bronchoscope, which was released in 2018, has a wider up-angulation range, which is theoretically preferable to previous models for puncturing the subaortic lesions. We report herein two cases with subaortic lesions that could not be evaluated using a conventional ultrasound bronchoscope, but that were successfully evaluated and diagnosed via EUS-B-FNA employing a new ultrasound bronchoscope.
\end{abstract}

Keywords: Bronchoscopy; case report; endobronchial ultrasound; endoscopic ultrasound; lung cancer; subaortic lesion

Submitted Sep 23, 2019. Accepted for publication Nov 28, 2019.

doi: $10.21037 /$ tlcr.2019.12.04

View this article at: http://dx.doi.org/10.21037/tlcr.2019.12.04

\section{Introduction}

Transesophageal endoscopic ultrasound with bronchoscopeguided fine needle aspiration (EUS-B-FNA) using an endobronchial ultrasound (EBUS) bronchoscope has been reported to be useful for diagnosing mediastinal lesions (1) and for mediastinal staging of lung cancer in combination with endobronchial ultrasound-guided transbronchial needle aspiration (EBUS-TBNA) (2,3). In 2018, a thirdgeneration Olympus EBUS bronchoscope (BF-UC290F; Olympus, Tokyo, Japan, Figure $1 A$ ) was launched. It has a $40^{\circ}$ wider up-angulation range of the bending section and a $5^{\circ}$ steeper needle angle compared to a conventional secondgeneration EBUS bronchoscope (BF-UC260FW; Olympus; Figure $1 B$ ). The new EBUS bronchoscope is preferable for evaluating previously difficult-to-reach lesions by EUSB-FNA, including subaortic lesions. Here, we present two cases in accordance with the CARE-Guidelines (4).

\section{Case presentation}

Case 1

A 70-year-old woman was referred to our hospital to determine the cause of left recurrent nerve paralysis. She complained of hoarseness to the referring otolaryngologist and had been given a diagnosis of left recurrent nerve paralysis. She was a never-smoker with no medical history. In our hospital, a chest X-ray and computed tomography were taken, and revealed a tumor in the left upper lobe lung and an enlarged subaortic lymph node measuring $25 \mathrm{~mm}$ in shortest diameter (Figure 2A).

After informed consent was obtained, EUS-B-FNA through the esophagus was performed under local anesthesia and conscious sedation in the supine position for staging and diagnostic purposes. First, a conventional EBUS bronchoscope (BF-UC260FW; Figure 1B) was advanced through the esophagus, and the subaortic lymph node 

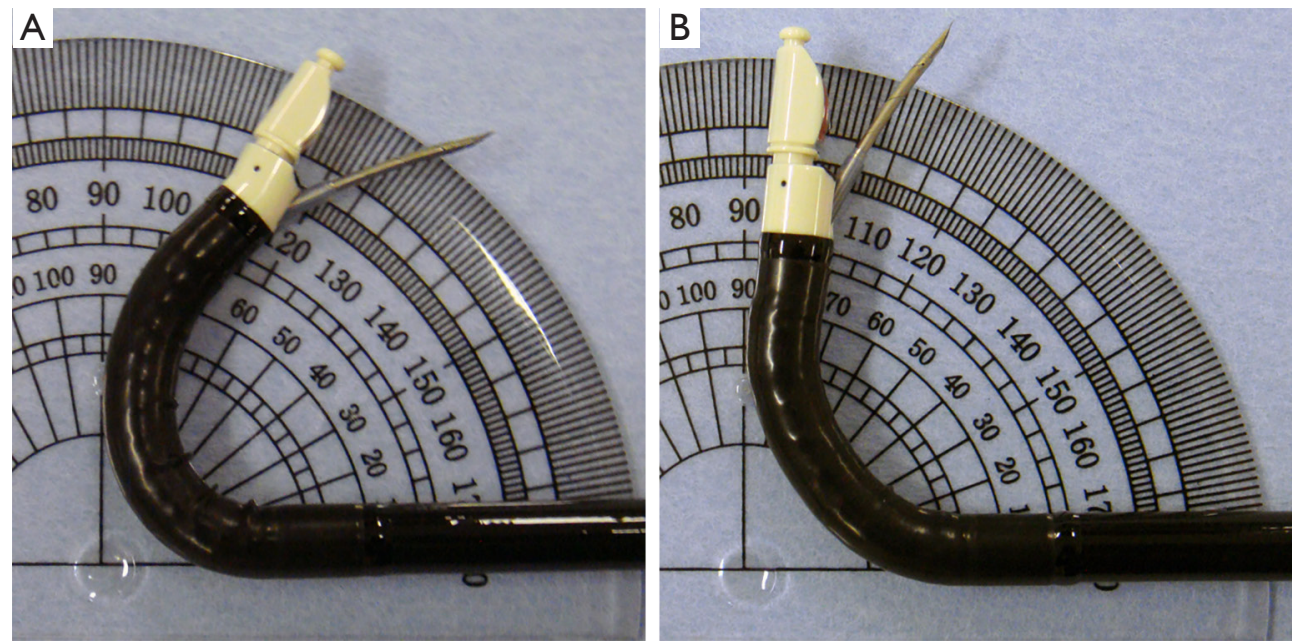

Figure 1 Comparison of the new ultrasound bronchoscope and the conventional ultrasound bronchoscope. (A) New ultrasound bronchoscope (BF-UC290F; Olympus) with a 19-gauge needle bent to the limit. (B) Conventional ultrasound bronchoscope (BF-UC260FW; Olympus) with a 19-gauge needle bent to the limit.
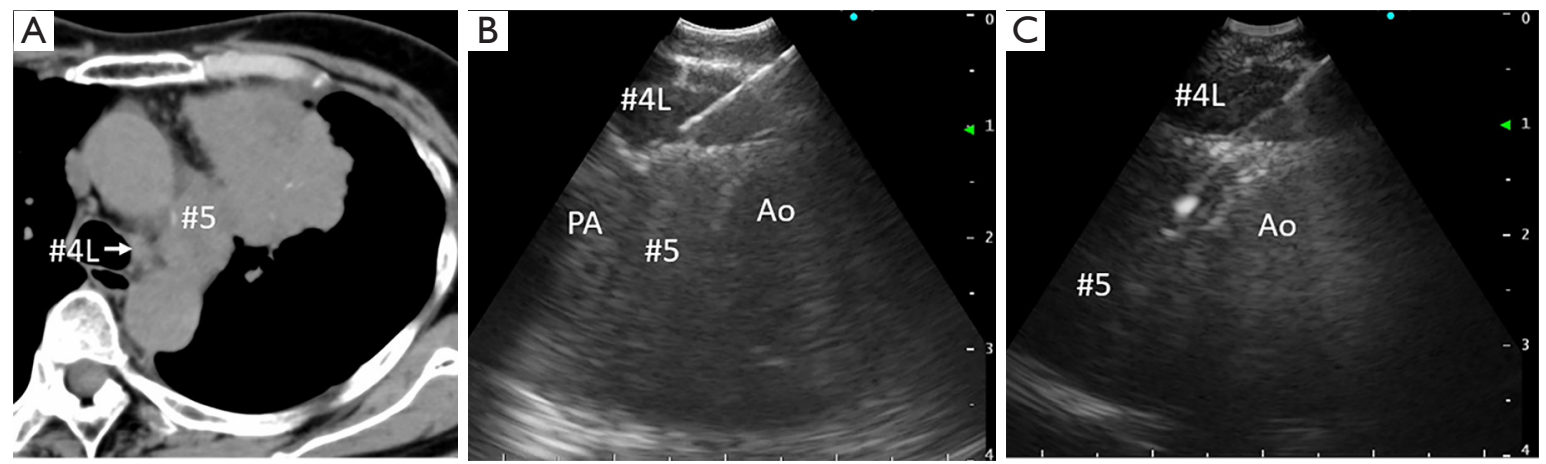

Figure 2 Chest computed tomography image and ultrasound images of Case 1. (A) Chest computed tomography image. (B) Ultrasound image acquired during endoscopic ultrasound with bronchoscope-guided fine needle aspiration (EUS-B-FNA) using a conventional ultrasound bronchoscope and a 19-gauge needle. (C) Ultrasound image acquired during EUS-B-FNA using the new ultrasound bronchoscope and a 19-gauge needle. \#4L, left lower paratracheal lymph node; \#5, subaortic lesion; Ao, aortic arch; PA, pulmonary artery.

was observed endosonographically. We tried to perform a needle puncture using a 19-gauge needle (ViziShot 2 Flex; Olympus); however, the needle only reached the left lower paratracheal lymph node and not the subaortic lymph node (Figures 2,3). The result of rapid on-site cytological evaluation (ROSE) using a quick staining method (DiffQuik; Kokusaishiyaku; Kobe, Japan) was negative. Then, we changed the conventional EBUS bronchoscope to the new EBUS bronchoscope (BF-UC290F; Figure 1A) and attempted a needle puncture in the subaortic lymph node. The needle reached the subaortic lymph node easily (Figures 2C,3), and the result of ROSE was positive after the first puncture. A total of four passes were performed to obtain an adequate quantity of histological material. There were no complications. She was given a final diagnosis of adenocarcinoma according to immunohistochemical staining of histological specimens obtained from the subaortic lymph node by EUS-B-FNA.

\section{Case 2}

A 63-year-old man with no symptoms and no relevant medical history, but who was a current smoker, underwent a medical examination; the chest $\mathrm{X}$-ray revealed an enlarged mediastinum. Chest computed tomography revealed a subaortic lesion $38 \mathrm{~mm}$ in the shortest diameter (Figure 4A); 
this was hypermetabolic on positron emission tomographycomputed tomography (Figure 4B). EBUS-TBNA was performed using a conventional EBUS bronchoscope (BF$\mathrm{UC2} 60 \mathrm{FW}$ ), but the result was non-diagnostic; the patient was referred to our hospital for examination of the subaortic lesion. After obtaining written informed consent, EUS-BFNA was performed using the new EBUS bronchoscope (BF-UC290F) and a 19-gauge needle, as described above for Case 1 (Figure 4C). ROSE was positive at the third

\section{: AME}

Video 1. An ultrasound video of the evaluation of

a case of lung adenocarcinoma with subaortic

lymphadenopathy '

via EUS-B-FNA usi

bronchoscope, 覆 1 is successfully

evaluated and di:

ultrasound bronchoscope

Masahide Oki ${ }^{\star}$, Hideo Saka, Yoshihito Kogure

Department of Respiratory Medicine, National

Hospital Organization Nagoya Medical Center Nagoya, Japan

Figure 3 An ultrasound video of the evaluation of a case of lung adenocarcinoma with subaortic lymphadenopathy that could not be diagnosed via EUS-B-FNA using a conventional ultrasound bronchoscope, but that was successfully evaluated and diagnosed employing a new ultrasound bronchoscope (5). EUS-B-FNA, endoscopic ultrasound with bronchoscope-guided fine needle aspiration.

Available online: http://www.asvide.com/watch/33040 puncture and a total of five passes were performed. There were no complications. Immunohistochemical staining of the histological specimens obtained via EUS-B-FNA was suggestive of metastatic adenocarcinoma.

The timelines of Cases 1 and 2 are shown in Figure 5.

\section{Discussion}

The combination of EBUS-TBNA and endoscopic ultrasound-guided fine needle aspiration (EUS-FNA) is recommended for mediastinal staging of lung cancer, and EUS-B-FNA is considered an alternative to conventional EUS-FNA $(2,3)$. The reported diagnostic utility of EUS-BFNA is comparable to that of conventional EUS-FNA (3); however, conventional EUS-FNA has an advantage of superior ability to access subaortic lesions. The dedicated EUS endoscope has strong bending power that is not easily affected by needle stiffness, a wide bending angulation range $\left(130^{\circ}-150^{\circ}\right.$ up-angulation), and a needle elevator that can be used to adjust the needle puncture angle. On the other hand, the bending power of the previous EBUS bronchoscope is insufficient for flexing the bronchoscope when a stiff needle is used; the bending capability is only $120^{\circ}$ up-angulation, and the protruding needle angle is nonadjustable and gentle. Thus, several investigators have reported good accessibility of EUS-FNA using a dedicated EUS endoscope for subaortic lymph nodes (6), while only a few cases of successful evaluation of subaortic lymph nodes by EUS-B-FNA have been reported $(7,8)$.
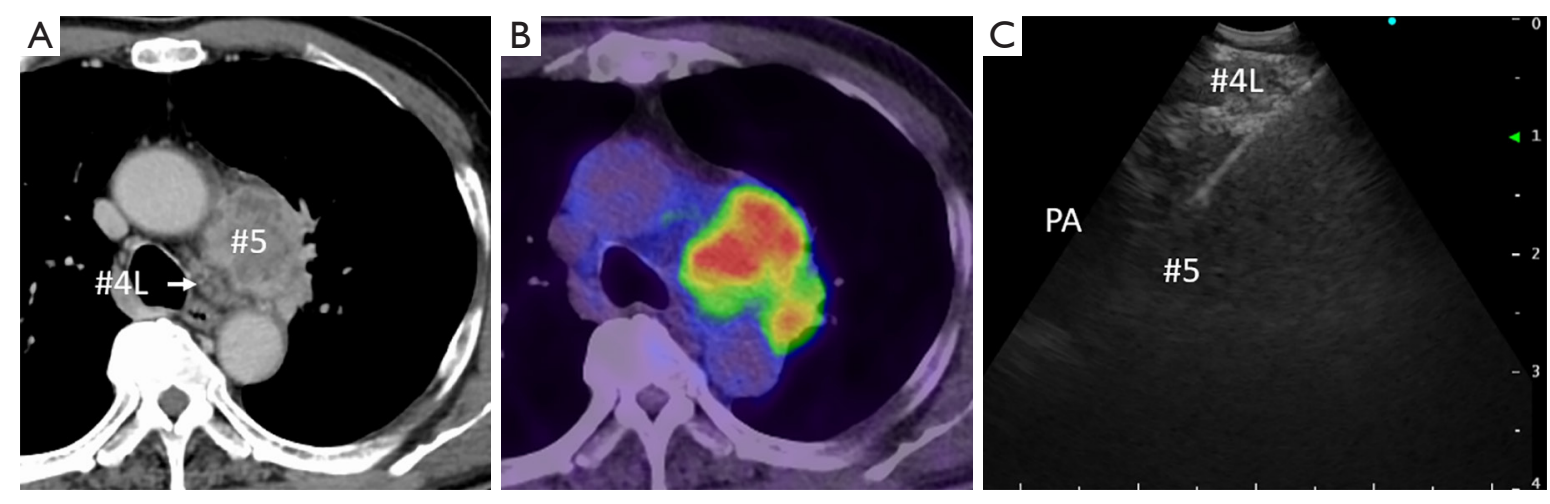

Figure 4 Chest computed tomography image, positron emission tomography-computed tomography image and ultrasound image of Case 2. (A) Chest computed tomography image. (B) Positron emission tomography-computed tomography image. (C) Ultrasound image acquired during endoscopic ultrasound employing bronchoscope-guided fine needle aspiration using a new ultrasound bronchoscope and a 19-gauge needle. \#4L, left lower paratracheal lymph node; \#5, subaortic lesion; PA, pulmonary artery. 
Case 1

Weeks

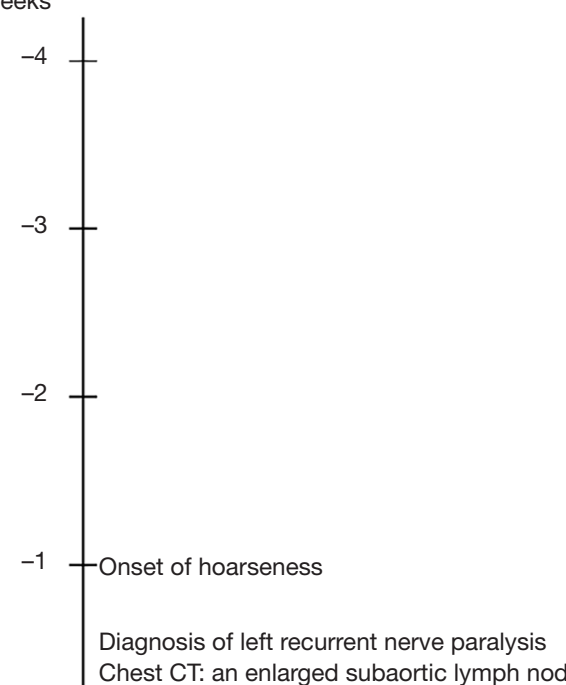

EUS-B-FNA using a conventional EBUS bronchoscope (ROSE-negative)

0

-

-followed by the new EBUS bronchoscope (ROSE-positive)

Pathological diagnosis of adenocarcinoma only in samples obtained

+1 with the new EBUS bronchoscope

$+2$

Figure 5 Timelines of Cases 1 and 2. CT, computed tomography; EBUS-TBNA, endobronchial ultrasound-guided transbronchial needle aspiration; EUS-B-FNA, endoscopic ultrasound with bronchoscope-guided fine needle aspiration; PET-CT, positron emission tomographycomputed tomography; ROSE, rapid on-site cytological evaluation.

Since the first generation of EBUS bronchoscopes was launched in 2004, EBUS bronchoscopes and dedicated EBUS needles have improved, enabling better access to mediastinal regions through the airway and esophagus. The new EBUS bronchoscope has an up-angulation range of $160^{\circ}$, and the protruding needle angle is $5^{\circ}$ steeper than that of previous EBUS bronchoscopes. In addition, the 19-gauge EBUS needle used in these cases was more flexible than the thinner 21- and 22-gauge EBUS needles. Although still technically challenging, the combined use of a new EBUS bronchoscope and a flexible EBUS needle may provide greater opportunity to evaluate subaortic lesions.

\section{Limitations}

This study was performed using only two cases; further
Case 2

Weeks

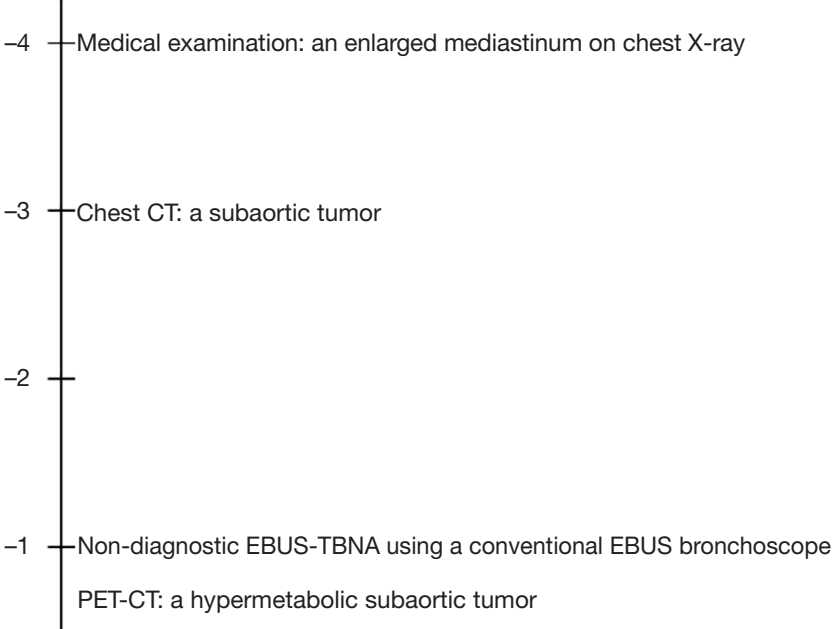

0 -EUS-B-FNA using the new EBUS bronchoscope (ROSE-positive)
$+2$

Pathological diagnosis of metastatic adenocarcinoma large-scale studies are necessary to elucidate the usefulness of EUS-B-FNA with the new EBUS bronchoscope for evaluating subaortic lesions.

\section{Acknowledgments}

None.

\section{Footnote}

Conflicts of Interest: The authors have no conflicts of interest to declare.

Ethical Statement: The authors are accountable for all aspects of the work in ensuring that questions related to the accuracy or integrity of any part of the work were 
appropriately investigated and resolved. Informed consent for publication was obtained in writing from Cases 1 and 2 .

\section{References}

1. Oki M, Saka H, Ando M, et al. Transbronchial vs transesophageal needle aspiration using an ultrasound bronchoscope for the diagnosis of mediastinal lesions: a randomized study. Chest 2015;147:1259-66.

2. Vilmann P, Clementsen PF, Colella S, et al. Combined endobronchial and esophageal endosonography for the diagnosis and staging of lung cancer: European Society of Gastrointestinal Endoscopy (ESGE) Guideline, in cooperation with the European Respiratory Society (ERS) and the European Society of Thoracic Surgeons (ESTS). Endoscopy 2015;47:545-59.

3. Korevaar DA, Crombag LM, Cohen JF, et al. Added value of combined endobronchial and oesophageal endosonography for mediastinal nodal staging in lung cancer: a systematic review and meta-analysis. Lancet Respir Med 2016;4:960-8.

4. Riley DS, Barber MS, Kienle GS, et al. CARE guidelines

Cite this article as: Oki M, Saka H, Kogure Y. Transesophageal needle aspiration using a third-generation Olympus ultrasound bronchoscope for subaortic lesions: a report of two cases. Transl Lung Cancer Res 2019;8(6):1152-1156. doi: 10.21037/ tlcr.2019.12.04 for case reports: explanation and elaboration document. J Clin Epidemiol 2017;89:218-35.

5. Oki M, Saka H, Kogure Y. An ultrasound video of the evaluation of a case of lung adenocarcinoma with subaortic lymphadenopathy that could not be diagnosed via EUS-BFNA using a conventional ultrasound bronchoscope, but that was successfully evaluated and diagnosed employing a new ultrasound bronchoscope. Asvide 2019;6:355. Available online: http://www.asvide.com/watch/33040

6. Liberman M, Sampalis J, Duranceau A, et al. Endosonographic mediastinal lymph node staging of lung cancer. Chest 2014;146:389-97.

7. Hwangbo B, Lee GK, Lee HS, et al. Transbronchial and transesophageal fine needle aspiration using an ultrasound bronchoscope in mediastinal staging of potentially operable lung cancer. Chest 2010;138:795-802.

8. Oki M, Saka H, Ando M, et al. Endoscopic ultrasoundguided fine needle aspiration and endobronchial ultrasound-guided transbronchial needle aspiration: Are two better than one in mediastinal staging of non-small cell lung cancer? J Thorac Cardiovasc Surg 2014;148:1169-77. 